\title{
El embarazo no planificado en la adolescencia ¿es posible un diálogo interdisciplinar?
}

Victoria Keller
IIEGE/UBA

El embarazo en la adolescencia es un tema que ha sido extensamente estudiado desde diferentes disciplinas; la sociología y la antropología, la epidemiología y la salud pública, la economía y la historia. Asimismo, ha concentrado el interés de múltiples agencias de cooperación internacional, ONG laicas y confesionales, Banco Mundial y, últimamente en la región, estados nacionales con sus planes específicos de prevención y abordaje (México, Colombia, la Argentina, entre otros).

En cada una de sus justificaciones, el interés por el tema adopta una relevancia máxima, aunque no siempre por los mismos motivos; desde la salud pública, porque las adolescentes que se convierten en madres tienen peores indicadores de salud perinatal (especialmente las menores de quince años); desde la economía, porque las complicaciones de un embarazo representan un mayor gasto de salud y porque una adolescente que se convierte en madre sin antes haber completado sus estudios genera un gasto público mayor; desde la sociología y la antropología, por el estigma con el que cargan las madres jóvenes; desde las ONG confesionales, por el tipo de cuidado que podrán brindar a sus hijos, y desde la epidemiología con perspectiva de género, por la alta proporción de no planificación del embarazo, que evidencia el no acceso a métodos anticonceptivos de este grupo etario.

Ante este panorama, ¿es posible establecer un diálogo entre las disciplinas? ¿Puede la abundancia de estadísticas vitales y datos epidemiológicos vincularse con los hallazgos cualitativos?

Dada la multicausalidad del embarazo en la adolescencia, es metodológicamente imposible establecer causalidades discretas, razón por la cual se suele hablar de factores asociados que suelen estar estandarizados en modelos ecológicos que separan el nivel normativo del comunitario, familiar y social. Estos modelos, privilegiados por las agencias de cooperación internacional y, por ende, por la salud pública, suelen ser insuficientes, por un lado, en su dimensión histórica y, por el otro, en las particularidades culturales y en las motivaciones más íntimas de las sujetas, con sus inasibles categorías de deseo y sexualidad que tanto eluden los estudios cuantitativos. El embarazo, como el suicidio que describió Durkheim, es fascinante porque reviste una dimensión personal y otra fuertemente social, al punto de mostrar su regularidad en las estadísticas vitales de los últimos treinta años (2.500-3.000 partos por año en niñas/adolescentes menores de quince años, y 80.000-110.000 partos por año en adolescentes entre quince y diecinueve años). Si bien el grupo etario 15-19 tuvo mayores oscilaciones en las últimas décadas, la tasa de fecundidad se mantuvo 
entre un 60/1.000 durante tres décadas, hasta alcanzar su punto más bajo en el último año registrado, 2017, cuando llegó a un 53,1/1.000. ¿Qué motiva este descenso de los últimos tres años? Solo podemos aventurar hipótesis.

El dato del 64\% de no planificación que muestra el SIP (Sistema Informático Perinatal), aunque cuestionada la calidad de su registro, es revelador en tanto se repite en todos los países del Cono Sur. Nos convoca entonces como feministas porque nos habla de una enorme cantidad de adolescentes (y de mujeres adultas también, que son el 50\%) que se convierten en madres - sus vidas cambiadas irreversiblemente, sus cuerpos y sus futuros comprometidos- sin haberlo planificado. Estudios más específicos diferencian los embarazos deseados pero no planificados de los no deseados pero asumidos, y de los no planificados y no asumidos. A nivel estadístico, no sería posible construir este dato pero sí tal vez podamos asirnos de los estudios cualitativos para entender un poco mejor este indicador.

¿Qué se construyó desde la sociología y la psicología social? Desde la sociología, y más preocupados por las trayectorias educativas y el carácter expulsivo del sistema educativo hacia estas adolescentes, gran parte de la investigación social se centró en analizar las motivaciones de las adolescentes de sectores populares para convertirse en madres. Estos estudios, previos a las sanciones de las leyes de ESI y de la creación del Programa de Salud Sexual, exploraron el significado de la maternidad en sectores populares, mientras que, tal vez involuntariamente, reforzaban hipótesis culturalistas acerca del embarazo en la adolescencia en estos sectores socioeconómicos. El problema de este enfoque es que si bien tenía una mirada en las barreras estructurales en el ejercicio de los derechos sexuales y reproductivos, asumía que los embarazos en la adolescencia de los sectores populares eran más deseados que los de los sectores medios.

La cultura, una vez más, era interpretada de la manera que menos nos interesa a les antropólogues; como una dimensión estática e inalterable de la condición humana. Peor aún: reificada como el último reducto de dignidad de los más vulnerables, prístina e impoluta de intervención estatal. En este punto, este tipo de literatura se entrelaza con el modelo médico hegemónico que primó (y continúa vigente en muchos espacios) en la Argentina en su enfoque paternalista y pronatalista.

En este sentido, el factor de no intencionalidad del embarazo aporta herramientas muy valiosas para entender el embarazo adolescente desde los datos estadísticos. Un estudio en Latinoamérica que analizó la no intencionalidad según nivel socioeconómico encontró que las adolescentes de los quintiles más bajos de la sociedad presentan los mismos porcentajes de no deseabilidad que sus pares más acomodadas, con lo cual la explicación debe buscarse en otro lugar: "Dado que las diferencias observadas en la fecundidad adolescente no se deben a mayores preferencias reproductivas y que la fecundidad no deseada es alta también entre los pobres, entonces el factor diferenciador ha de ser el acceso a anticonceptivos, respecto del cual las pobres tendrían muchas más barreras". ${ }^{1}$

1 Rodríguez Vignoli, J. (2017). Fecundidad no deseada entre las adolescentes latinoamericanas. Un aumento que desafía la salud sexual y reproductiva y el ejercicio de derechos. CEPAL/ UNFPA. 


\section{La "anomalía" estadística}

¿Por qué no desciende la tasa de fecundidad adolescente en Latinoamérica? Analistas cuantitativos quedan desconcertados ante el fenómeno que llaman "la anomalía latinoamericana"; en una región donde el PBI per capita crece, donde la tasa global de fecundidad (cantidad de hijos por mujer entre 15-49 años) desciende, donde los avances en el ingreso y la permanencia en el sistema educativo (poderoso factor protector para el embarazo adolescente) reflejan una tasa bruta de matrícula alta, no parece posible postergar el primer hijo. Esta anomalía pone a la región en paralelo con África, la única otra región del mundo que no ha podido bajar el indicador.

Sin hacer menoscabo de las variables macroeconómicas que estructuran la coyuntura de nuestras sociedades al profundizar las iniquidades sociales, tal vez debamos mirar hacia otro nivel menos estructural, no obstante más decisivo, para el ejercicio de los derechos sexuales y los derechos no reproductivos de las adolescentes; el acceso a la información, a los métodos anticonceptivos modernos y a la interrupción del embarazo en condiciones seguras. Y con ello, debemos tener una mirada crítica hacia el abordaje con que el sistema de salud y el sistema educativo tratan a les adolescentes, que está menos actualizado en derechos y evidencia científica y más enraizado en creencias de lo que se puede suponer. Para esto existe un numeroso corpus bibliográfico que señala cómo debe ser este acceso para una correcta adopción y adhesión a métodos anticonceptivos. Las políticas sanitarias de este país han sabido interpretar esta literatura y adaptarla en diferentes documentos acerca de consejerías en salud sexual y adecuación de espacios de salud para la atención a adolescentes que, entre otras cosas, incluyen de qué manera y cuáles métodos anticonceptivos ofrecer (de barrera, de larga duración, hormonales), el tipo de escucha necesaria, los ejes de género y derechos, etc. Queda un largo camino por recorrer en términos de lograr una efectiva adecuación de los equipos de salud para garantizar el acceso a métodos de todes les adolescentes, en todas las provincias del país.

Tal vez el descenso de los últimos tres años de la tasa de fecundidad 15-19 se deba a un proceso de consolidación acumulada de estas políticas en los ámbitos nacionales y provinciales, tal vez a la inclusión del implante subdérmico en la canasta de métodos anticonceptivos desde 2014, tal vez a las paulatinas adaptaciones del fallo FAL en la mayoría de las normativas provinciales, tal vez a la marea feminista que rápidamente inunda de verde las escuelas y los centros de salud; no hay forma metodológicamente válida de dar con una respuesta acabada a este cambio en el indicador. Lo que sí sabemos es que la permanente investigación acerca de qué esperan y qué respuesta obtienen les adolescentes del sistema de salud y del educativo debe guiar el cumplimiento de sus derechos sexuales y las políticas que lo garantizan. 
